\title{
Une poétique des espaces : lecture écocritique des géographies et langages hybrides dans Verre Cassé
}

\author{
Nathan Germain \\ Providence College
}

Dans le roman Verre Cassé d'Alain Mabanckou, une autre réalité de l'Afrique est présentée. À travers les écrits du personnage éponyme, le lecteur entre dans la vie quotidienne d'un quartier pauvre à Pointe-Noire, capitale économique de la République du Congo (- Brazzaville). Le livre donne une voix à cet espace normalement muet, mais il ne se contente pas d'être le porte-parole de ces peuples impuissants. Dans un langage qui est toujours à mi-chemin entre le français populaire et le français littéraire, Verre Cassé représente une manière de tenir compte de plusieurs espaces, tant au niveau global que local. C'est cette double opération - à la fois une prise de conscience d'un espace particulier et une ouverture plus générale au monde - qui nous intéressera dans le cadre de cette étude.

Nous nous demanderons en particulier quel rôle joue le langage dans ce voyage entre des espaces du monde. Verre Cassé semble nous donner un moyen d'existence «entre-deux »: entre les français populaire et littéraire, entre une prise de conscience locale et une réflexion littéraire plus globale. Une analyse soignée de la langue et du langage sera donc au cœur de notre projet. Nous examinerons le rapport entre une géographie environnante (le bar ou le quartier) et une géographie planétaire (la France, l'Afrique, et le monde) dans Verre Cassé, surtout à travers l'influence du langage. Dans quelle mesure peut-on dire que le langage permet un ancrage et une ouverture en même temps? À partir de quelques notions de base, proposées par des géographes (Dardel et Berque) et un anthropologue (Augé), nous examinerons le roman de Mabanckou comme une poétique des espaces. Enfin, nous élargirons la portée de l'étude en analysant les théories de Glissant ("Tout-monde», "Relation») pour voir dans quelle mesure Mabanckou participe à cette vision ouverte «à la rumeur du monde » (Le monde 182). Enfin, pouvons-nous dire que Mabanckou crée une littérature nouvelle et innovatrice, qui «nous convie à être nous-mêmes sans nous fermer à l'autre » (Le monde 64), une littérature qui s'ouvre au monde, grâce au langage? Ce sont les grandes questions qui nous guideront tout au long de cette étude.

\section{L'espace et la littérature}

1 Nous nous référerons à cette édition du livre (nous mettrons les références aux pages entre parenthèses) : Alain Mabanckou, Verre Cassé, Paris, Seuil, 2005. 
Avant d'entamer notre étude strictement littéraire, il faut considérer quelques concepts géographiques et anthropologiques qui aideront à mieux comprendre l'importance de l'espace dans le roman. Dans son étude géographique, L'Homme et la Terre, Éric Dardel affirme que : «La réalité géographique, pour l'homme, c'est d'abord là où il est, les lieux de son enfance, l'environnement qui le convoque à sa présence. Des terres qu'il foule ou qu'il laboure, l'horizon de sa vallée, ou bien sa rue, son quartier, ses déplacements quotidiens à travers la ville » (46). Dardel est l'un des premiers géographes qui cherchent à réunir les disciplines de la géographie et de la philosophie (surtout la philosophie de l'existence). Pour Dardel, la géographie n'est pas une science «indifférente ou détachée », au contraire, la géographie c'est « ce qui [...] m’intéresse au plus haut degré » (46), c'est-à-dire les attaches existentielles entre la terre et l'être humain qu'il appelle la " géographicité de l'être ». Dans le roman Verre Cassé, le lecteur entre dans une de ces géographicités qui est celle d'un quartier à Pointe-Noire. Le protagoniste qui s’appelle Verre Cassé établit les liens existentiels avec sa réalité géographique et il essaie de communiquer ces attaches complexes à son lecteur. Déjà, d'après la définition de Dardel, nous voyons que l'idée de l'espace est d'une importance à la fois géographique et philosophique.

Cependant la définition proposée par Dardel n'est pas satisfaisante en ce qu'elle ne permet pas de décrire toute la complexité des rapports entre les êtres humains et leur environnement. La « réalité » d'un milieu n'est pas seulement matérielle ou spatiale, elle est aussi définie par un réseau de relations sociales, éthiques, culturelles, écologiques, et linguistiques. Augustin Berque donne le nom d'«écoumène» à cet ensemble de relations: "L'écoumène est une relation : la relation à la fois écologique, technique, et symbolique de l'humanité à l'étendue terrestre. Elle ne se borne donc pas à la matérialité de l'étant physique » (14). Berque est un géographe qui voit aussi le lien entre l'existence et la géographie comme fondamental à la connaissance : "l'être humain est géographique [...] il est d'abord, et nécessairement, déterminé par une certaine relation (géographique) [...] Cette relation fonde notre humanité même; elle en est la condition» (10-12). La définition de Berque arrive mieux à prendre en compte la multitude de relations qui existent entre l'humanité et la terre. Le concept d'« écoumène » est donc un bon fondement pour aborder notre étude du langage dans Verre Cassé. Il est également important de constater que la relation envisagée par Berque ne se limite pas à notre géographie locale: «L'écoumène est donc à la fois mesurable et incommensurable. Tout comme la terre, à l'horizon, se conjoint au ciel, notre être s'étend au-delà du bout de nos doigts, pour atteindre les antipodes, la planète Mars, et toujours plus loin encore, jusqu'aux confins de l'Univers » (14). Verre Cassé, comme nous verrons, cherche 
à chanter le singulier de son espace tout en explorant une communion plus large et profonde, une ouverture aux relations ontologique et géographique.

Or, dans cette définition de l'écoumène, il ne serait pas difficile de courir le risque de l'idéal universel qui effacerait la particularité, la différence. En effet, la disparition du singulier est peut-être l'un des phénomènes les plus importants de la modernité, surtout dans le contexte de la mondialisation; une modernité que l'anthropologue Marc Augé définit comme phénomène qui crée des espaces sans symbolisme ${ }^{2}$. Berque, il faut en convenir, a déjà anticipé ce problème, dans son concept de "l'universion », la réduction du divers au même : «L'histoire montre effectivement que l'universion comporte souvent l'élimination d'autrui, selon une échelle allant de la violence symbolique envers les déviants jusqu'au génocide massif » (Berque 66). Dans la vie moderne, nous reconnaissons les signes de cette élimination du divers dans la prolifération des espaces «banaux » : hôpitaux, cliniques, aéroports, métros, banques, grandes surfaces, autant de "non-lieux » (Augé 100) reproduits à l'infini "qui ne sont pas euxmêmes des lieux anthropologiques » (Augé 100). Le non-lieu est un espace sans symbolisme, sans écoumène (Augé 100-105). Cela dit, nous constatons que le roman Verre Cassé est un texte tout à fait original sur ce point. Le roman parvient à chanter la particularité d'un lieu (le contraire du non-lieu) tout en dialoguant avec un espace plus large : l'universel. Nous examinerons plus tard comment Mabanckou parvient à engager l'universel sans effacer le divers.

Enfin, il faut considérer l'espace comme l'un des thèmes les plus souvent repris dans la littérature. La littérature « nous transporte en imagination dans des contrées inconnues qu'elle nous donne un instant l'illusion de parcourir et d'habiter ", comme le note Gérard Genette (43). Cette appréciation pour le pouvoir évocateur de la littérature fait partie aussi de l'expérience personnelle de Verre Cassé : « disons que j'ai plutôt voyagé sans bouger de mon petit coin natal, j'ai fait ce que je pourrais appeler le voyage en littérature, chaque page d'un livre que j'ouvrais retentissait comme un coup de pagaie au milieu d'un fleuve, je ne rencontrais alors aucune frontière au cours de mes odyssées » (170-1). Nous pouvons alors constater que l'espace est un fondement essentiel et pour la littérature et pour le projet littéraire de Verre Cassé ${ }^{3}$. La particularité de l'espace littéraire est sa capacité de mettre en relation plusieurs espaces normalement éloignés, tant physiquement que symboliquement.

\footnotetext{
2 Voir à ce propos l'ouvrage de Marc Augé, Non-lieux : Introduction à une anthropologie de la surmodernité, Seuil, 1992.

${ }^{3}$ Les rapports espace-littérature-langue sont analysés par Frédéric Mambenga-Ylagou (2007) qui leur donne le nom de "géographicité de la langue et de l'imaginaire ». Ceux-ci constituent l'un des éléments essentiels de la littérature africaine francophone de nos jours.
} 
La géocritique, une approche théorique en études littéraires associée à son initiateur, Bertrand Westphal, se propose d'analyser «l'ensemble de l'étude des espaces humains en littérature, ou mieux: l'étude des espaces humains appréhendés dans leur globalité » («Pour une approche »12) ${ }^{4}$. Cette approche met l'accent sur un double rôle de l'espace en littérature, à savoir que la littérature ne se contente pas de représenter l'espace, elle contribue également à la manière dont les êtres humains perçoivent, imaginent, et construisent l'espace. Selon Westphal, une étude proprement géocritique se doit de proposer quatre méthodes essentielles : le géocentrisme ${ }^{5}$, ou l'espace comme élément central de l'analyse; la "multifocalisation », la multiplication des perspectives et savoirs qui portent sur le lieu étudié; la " polysensorialité », ou la mobilisation de toutes les sensations pour appréhender un lieu; et la "stratigraphie », c'est-à-dire l'attention portée à l'accumulation des couches temporelles de la mémoire culturelle (La géocritique : réel, fiction, espace 200). La géocritique s'inscrit donc dans le « tournant spatial ${ }^{6}$ des études littéraires et des sciences sociales. Elle offre de nombreux outils utiles à l'édification d'une compréhension globale du fonctionnement de l'espace dans la littérature et même dans nos vies. Pour Westphal, il est temps de :

songer à articuler la littérature autour de ses relations à l'espace, de promouvoir une géocritique, poétique dont l'objet serait non pas l'examen des représentations de l'espace en littérature, mais plutôt celui des interactions entre espaces humains et littérature, et l'un des enjeux majeurs une contribution à la détermination/indétermination des identités culturelles (« Pour une approche»17).

Le géocritique Eric Prieto suit de près cet appel, tout en souhaitant un approfondissement par l'intersection entre la géocritique et l'écocritique. Pour Prieto (2014), il s'agit de complémenter l'analyse textuelle des espaces en littérature propre à la géocritique avec l'importance que l'approche écocritique accorde à la place humaine au sein du monde biophysique comme expérience incarnée matériellement, afin de nous aider à prendre conscience des modes de représentation écologiquement responsables et à mieux comprendre notre existence en tant que relation. Pourquoi lire Mabanckou d'une manière écocritique? Cette approche théorique en études littéraires et culturelles explore les relations diverses entre les êtres humains et les environnements dont ils font partie. La théorie et la pratique de l'écocritique se sont fortement développées depuis les années 1990, notamment aux États-Unis et dans le monde universitaire anglophone. Toutefois, l'approche commence à intéresser les

\footnotetext{
${ }^{4}$ Michel Collot, pour sa part, définit la « géocritique » comme approche qui étudie « les représentations de l'espace dans les textes eux-mêmes » (" Pour une géographie littéraire » par. 8, www.fabula.org/lht/8/collot.html).

${ }^{5}$ Le géocentrisme selon Westphal n’a rien à voir avec le géocentrisme pré-copernicien. Pour Westphal, il s'agit de mettre l'espace terrestre au cœur des préoccupations de la critique.

${ }^{6}$ Voir, par exemple, Robert T. Tally, Spatiality, Routledge, 2013.
} 
critiques littéraires français et ceux du monde francophone comme en témoigne le numéro spécial d'Écologie et Politique (2008) intitulé «Littérature et écologie : vers une écocritique ». Dans ce numéro, les contributeurs s'interrogent sur les relations entre conscience environnementale et esthétique littéraire. Or, les écocritiques français qui sont membres de l'Association for the Study of Literature and Environment (ASLE) sont, pour la plupart, des universitaires qui étudient la littérature anglo-américaine. ASLE a pris de l'ampleur dans monde depuis ses débuts, mais il ne reste aucune branche affiliée de l'association en France ou en Afrique ${ }^{7}$. Des critiques comme Stéphanie Posthumus et Ursula Heise ont déjà évoqué le déficit intellectuel dont une écocritique monolingue souffrirait (Posthumus 86).

Il est donc temps de remédier à ce problème en proposant des lectures écocritiques et géocritiques des écrivains d'expression française. La présente étude sur Verre Cassé d'Alain Mabanckou suit donc l'appel de l'écocritique Stéphanie Posthumus, qui propose une écocritique française qui est « provisoire, exploratrice, et originale » (86). L'analyse écocritique du roman Verre Cassé de Mabanckou participe pleinement à cet appel en étudiant la manière dont le roman met en œuvre une vision nouvelle sur l'espace en littérature à travers une hybridation originale du langage littéraire et géographique. Prenant en compte des perspectives diverses, de la géographie et de la géocritique française jusqu'aux théories de la relation d'Édouard Glissant en passant par les études postcoloniales francophones, cette étude cherche démontrer la pertinence des approches pluridisciplinaires dans la lecture des littératures contemporaines. Dans un monde qui se créolise, le texte de Mabanckou intervient en proposant une autre vision sur les relations géographiques, littéraires, et linguistiques qui tissent des liens insoupçonnés entre des espaces et des populations. Finalement, c'est à travers cette exploration de l'espace, du langage, et de leur hybridation que Mabanckou propose une nouvelle identité pour un monde en train de se créoliser, un acte de fusion à travers la littérature entre les mondes humain et naturel.

\section{Les deux espaces : l'ici et l'ailleurs}

\footnotetext{
${ }^{7}$ L'European Association for the Study of Literature, Culture, and Environment (EASLCE) est une association affiliée, mais son action comprend toutes les littératures et cultures européennes. La branche pan-européenne, l'European Association for the Study of Literature, Culture and Environment (EASLCE) ainsi que sa revue Ecozon@, dont la mission est de " refléter la richesse et diversité culturelles, linguistiques, et culturelles du continent européen », ont aidé à remédier aux problèmes de l'anglo-centrisme. Bien que la revue accepte des contributions en cinq langues, la majorité des articles sont publiés en anglais. Voir http://www.ecozona.eu/pour la revueEcozon@et https://www.easlce.eu/pour l'EASLCE.
} 
Il y a deux espaces dominants dans le roman que nous pourrions appeler «l'ici » et « l'ailleurs ». Dans l'ensemble du livre, « l'espace » le plus important est le bar «Le Crédit a voyagé »". C'est ici que Verre Cassé se voit confier par le patron la mission d'écrire l'Escargot entêté, et où il commence à écrire un cahier dans lequel il note les histoires des autres clients, ainsi que sa propre histoire. C'est l'Escargot entêté qui définit, le premier, le projet littéraire : « tu devrais écrire, je veux dire, écrire un livre [...] qui parlerait de nous ici $[. .$.$] de cet endroit unique$ au monde » (158). Or, la notion de l'espace « ici », l'espace local va s'étendre tout au long du roman. En même temps, Verre Cassé va chercher de plus en plus une communion avec d'autres espaces, avec «l'ailleurs». S'il communique souvent avec la France, son projet littéraire le met de plus en plus en relation avec d'autres espaces et auteurs africains, tout en entamant la conversation avec des espaces lointains situés aux quatre coins du monde.

Le livre, ou le cahier rend compte d'un espace particulier, à savoir le bar « Le Crédit a voyagé » dans le quartier Trois-Cents à Pointe-Noire. Bien que cet espace semble être un lieu de déchéance pour les alcooliques, un espace de rencontre pour tous les marginaux de la ville, le cahier représente quelque chose de bien plus positif pour Verre Cassé et pour toute une société. À travers les histoires interposées, nous assistons directement à la prise de parole d'une classe normalement muette et démunie de pouvoir et à la naissance d'un SDF alcoolique qui devient écrivain. Au début, le bar est un espace non défini, ou limité à sa fonction d'espace où s'échouent les laissés-pour-compte de la mondialisation, mais il devient rapidement un lieu singulier avec ses histoires, ses mythes, sa culture, sa société, son langage propre, et obtient même un statut littéraire grâce aux témoignages des clients réguliers et à l'écriture intertextuelle de Verre Cassé. L'espace du bar s'anime à travers les récits profondément ancrés dans la réalité sociale de cet espace « ici » du quartier Trois-Cents à Pointe-Noire, mais il n'en est pas pour autant dépourvu de relations avec des espaces «ailleurs ». Les clients racontent des histoires farfelues qui les relient à d'autres espaces. De plus, le jeu intertextuel fait voyager le texteur averti à travers l'Afrique et le monde entier. En effet, le nom même du bar, «Le Crédit a voyagé ", vient d'une pancarte derrière le comptoir dans un cabaret de Douala où Mabanckou a rencontré la journaliste camerounaise Suzanne Kala-Lobè, à l'occasion de l'opération « Portes d'Afrique » organisée par Le Figaro, une série d'« escales littéraires » qui visait à mettre en honneur douze ports du continent africain ${ }^{9}$. Assis au comptoir du cabaret, Mabanckou est fasciné par une pancarte portant l'inscription « Le crédit a voyagé », « belle association » (Mabanckou, Le

\footnotetext{
${ }^{8}$ En italiques dans le texte original.

${ }^{9}$ Voir Le monde est mon langage où Mabanckou raconte cette escale à Douala où il allait découvrir cette pancarte mystérieuse portant l'inscription « Le crédit a voyagé », à l'origine du nom du bar de son roman Verre Cassé (169-179).
} 
monde 176), selon l'écrivain congolais, de Mort à crédit et Voyage au bout de la nuit de Céline. Pour Mabanckou, la formule semble également résumer l'existence de ses personnages, autant de voyageurs aux lieux de naissance divers et aux destins géographiquement instables.

Verre Cassé fonctionne comme le scripteur de cet espace dont le patron se démarque de la tradition en constatant : «l'époque des histoires que racontait la grand-mère grabataire était finie, que l'heure était désormais à l'écrit parce que c'est ce qui reste », un patron qui rejette les formules du genre: "en Afrique quand un vieillard meurt, c'est une bibliothèque qui brûle » (11). Le patron se moque de l'oralité traditionnelle africaine (en citant l'écrivain malien Amadou Hampâté Bâ), ce qui est ironique parce que le style même du livre est inséparable de cette oralité. Cependant, on verra que la culture d'un espace est aussi préservée grâce à l'écriture qui permet de mélanger une langue mondiale (le français) avec le langage local pour produire une réaffirmation du lieu local et une ouverture au syncrétisme culturel.

Il est donc clair d'emblée que le livre ne met pas en scène une Afrique traditionnelle, mais plutôt des Afriques extrêmement urbaines et modernes. Le livre cherche à revaloriser la vie dans la ville, parfois en s'opposant farouchement à la vie au village ou «dans la brousse », où Verre Cassé refuse catégoriquement d'être renvoyé en disant : "j'ai dit non de manière ferme et irrévocable, je ne me voyais pas dans la brousse » (141-2) et il ne peut « pas du tout quitter la ville» pour la brousse qui représente «l'exil » et « l'arrière-pays » (146-7). Même si cette obstination lui coûte le poste d'instituteur, Verre Cassé est en train de réaffirmer la valeur de la vie en ville. Ce rapport étroit entre la cité et le citoyen constitue un exemple de rapport "écouménal », selon Berque. "Le mot cité nous vient de civitas, qui signifie d'abord "l'ensemble des citoyens" [...] civitas : à la fois un système symbolique, un ensemble humain, et un territoire avec ses traits matériels » (Berque 216-7). Tenter de chasser Verre Cassé hors de son quartier, de sa cité, c'est lui meurtrir l'être profond.

En restant dans la ville, Verre Cassé est libre de se réfugier au bar, où il assume son rôle de scripteur qui sauve l'espace de l'oubli. Comme tous les autres habitants du quartier Trois-Cents, il en fait partie intégrante, ils existent en relation étroite, "écouménale » avec le quartier, avec leur propre "écologie urbaine » que même les habitants de la grande Pointe-Noire ne comprennent pas. Ces Ponténégrins plus nantis «se demandaient alors comment les gens faisaient pour vivre en parfaite cohabitation avec les immondices, les mares d'eau, les carcasses d'animaux domestiques, les véhicules brûlés, la vase, la bouse, les trous béants des artères et les maisons qui étaient au bord de l'effondrement » (15). Verre Cassé fait l'énumération de la décrépitude, de la pourriture, dont le 
sens habituel se renverse totalement. Ce monde de la putréfaction devient un espace important, justement grâce à la relation symbiotique entre les habitants et l'espace.

Il y a plusieurs espaces « ailleurs » dans le livre, de la France aux États-Unis, de la «brousse» à Douala, à Cotonou, et à toute l'Afrique. Cet amas de références géographiques et littéraires empêche d'établir une hiérarchie de valeur, tant le lecteur se sent perdu dans un tourbillon éclectique où ni centre ni périphérie n'ont plus aucun sens. Il est pourtant clair que la France, tantôt raillée, tantôt vénérée, représente un ailleurs hautement important. Quelques clients du bar sont les gens qui ont « fait la France » comme, par exemple, l'Imprimeur : «je suis le plus important de ces gars parce que j'ai fait la France, et c'est pas donné à tout le monde, crois-moi ». La France est donc «l'unité de mesure, le sommet de la reconnaissance, y mettre les pieds, c'était s'élever au rang de ceux qui ont toujours raison » (54). Or, les histoires de ces personnages ayant « fait la France » posent des questions à plusieurs égards. D'abord, au niveau narratif, Verre Cassé se laisse emporter par le récit de chacun de ses concitoyens du bar, à tel point qu'on n'arrive plus à distinguer entre la voix de chaque conteur particulier et celle de l'auditeur/écrivain (Verre Cassé). Le lecteur partage alors la même expérience, le même transport vers un autre espace que le conteur. C'est une manière plus directe de dissoudre la frontière entre l'espace du bar et l'espace lointain, global. Dans un deuxième temps, les histoires racontent d'habitude un échec. Il ne faut pas comprendre cet échec comme une critique sévère de l'espace « ailleurs", mais comme un effort de rendre plus complexes et plus réels les rapports entre les espaces. Verre Cassé, qui se présente en scripteur fidèle, veut en finir avec les faux discours sur l'immigration réussie. En effet, la rencontre des «espaces" et des cultures s'avère chaotique, au sens productif donné par Glissant ${ }^{10}$. Par la suite, nous analyserons d'autres exemples de la mise en relief de ces espaces «ailleurs", en particulier par rapport au langage.

\section{Le langage et l'espace}

Tout langage est, de par sa nature, un espace composite où cohabitent plusieurs codes culturels, sociaux, et linguistiques. Tout énoncé est un tissu compliqué, avec des discours intercalés les uns à côté des autres. Même le langage particulier est un langage emprunté, car il est fondé sur des mots qu'on n'a pas inventés. Comme l'a dit Derrida dans Le monolinguisme de l'autre, « je n'ai

10 Cet état de « chaos » est examiné notamment par Glissant dans son Traité du Tout-monde: «J'appelle Chaos-monde le choc actuel de tant de cultures qui s'embrasent, se repoussent, disparaissent, subsistent pourtant, s'endorment ou se transforment, lentement ou à vitesse foudroyante » (Traité 22). 
qu'une langue, or ce n'est pas la mienne » (15). La coexistence langagière est même plus significative dans le contexte du roman africain francophone. Mabanckou en fait une des questions essentielles de son livre. Comme le dit Mambenga-Ylagou, l'hybridité langagière est l'un des éléments constitutifs du texte littéraire africain (135). C'est justement cette hybridité qui permet à Mabanckou de créer une nouvelle poétique des espaces, une poétique qui est enracinée dans l'espace urbain, mais qui reste consciente des autres espaces et discours et les accepte en même temps. Ce faisant, Mabanckou participe au renouveau du roman africain, selon Liana Nissim, qui identifie plusieurs motifs récurrents de cette nouvelle littérature :

[...] il s'agit de la présence dominante de l'univers urbain toujours cruel aux plus démunis, l'acceptation de l'hybridité culturelle, inséparable désormais d'une nouvelle identité qui mélange inextricablement modernité et passé traditionnel, le refus de l'écrivain de se borner à une fonction strictement politique et militante, au détriment de la création esthétique proprement dite (199-200).

Lisons d'abord les premières «phrases » du roman pour avoir une idée du langage employé par Verre Cassé ainsi que le caractère métalittéraire du roman : Disons que le patron du bar Le Crédit a voyagé m'a remis un cahier que je dois remplir, et il croit dur comme fer que moi, Verre Cassé, je peux pondre un livre parce que, en plaisantant, je lui avais raconté un jour l'histoire d'un écrivain célèbre qui buvait comme une éponge, un écrivain qu'on allait même ramasser dans la rue quand il était ivre, faut donc pas plaisanter avec le patron parce qu'il prend tout au premier degré [...] (11).

Notons d'emblée le style de ce petit passage qui commence in medias res sans majuscule. En effet, dépourvu de ponctuation (à part les virgules et les guillemets), le livre entier consiste en une seule phrase. Ce début et ce style indiquent que le livre est véritablement un cahier d'observations d'un espace qui n'a donc ni début ni fin. Le texte est déjà associé à la vie, qui, elle aussi, est un long processus continu. En plus, il faut remarquer le discours qui se rapproche du langage parlé. Le texte écrit du cahier est en quelque sorte une imitation des éléments lexicaux et syntaxiques de ce langage oral (bien que d'autres éléments comme l'intonation ne soient pas reproduits dans le texte écrit). En tant qu'instituteur, Verre Cassé décide même d'enseigner sa vision de la langue à ses élèves, signe d'une continuité langagière entre les générations :

Je jure aussi que j’aimais leur apprendre les participes passés conjugués avec l'auxiliaire avoir et qui s'accordent ou ne s'accordent pas selon qu'il fait jour ou nuit [...] et moi je leur disais que ce qui était important dans la langue française, c'était pas les règles, mais les exceptions, je leur disais que lorsqu'ils auraient compris et retenu toutes les exceptions de cette langue aux humeurs météorologiques les règles viendraient d'ellesmêmes $[\ldots]$ et qu'ils pourraient même se moquer de ces règles, de la structure de la phrase une fois qu'ils auraient grandi et saisi que la langue française n'est pas un long fleuve tranquille, que c'est plutôt un fleuve à détourner [...] (153). 
Voilà donc une apologie de l'exception langagière que Verre Cassé est en train de démontrer, une défense de la créativité et de l'importance du langage local.

Par sa forme, le roman est une série de citations, que ce soit celles des habitants ou des clients du bar ou que ce soit des citations littéraires. D'habitude, ces références littéraires ne sont pas signalées directement et parfois elles sont contrefaites ou détournées afin de masquer leur présence dans le texte. Très souvent, il est presque impossible de distinguer entre les discours populaires et littéraires :

[...] quelques vieux cons du quartier qui regrettaient la Case de Gaulle, la joie de mener une vie de boy, une vie de vieux Nègre et la médaille, une vie d'époque de l'exposition coloniale $[\ldots]$ et alors ces gens de bonne réputation ont tendu un piège sans fin au patron avec leurs casseurs cagoulés qui sont venus au milieu de la nuit, au cœur des ténèbres (14-15).

Dans ce passage, le lecteur érudit notera des références à quatre romans : Une vie de boy et Le vieux nègre et la médaille de Ferdinand Oyono, Un piège sans fin, d'Olympe Bhêly-Quenum, et Au caur des ténèbres de Joseph Conrad. Cette intégration du littéraire dans le populaire ne témoigne pas pour autant d'une volonté de vulgariser la littérature. Nous constatons plutôt que cette harmonisation entre les deux discours montre que même les peuples les plus démunis ont leur histoire à partager et elle est aussi littéraire que la grande littérature canonique. Ce mélange produit un langage unifié qui valorise les sphères littéraires et populaires en même temps. Or, comment croire que Verre Cassé écrirait d'une manière si intertextuelle si nous lisons son cahier au fur et à mesure de sa production? Il y a aussi une autre possibilité : les intertextes font partie intégrante de tout discours (même si ce discours est censé être oral, populaire, et sans préméditation) parce que nous vivons dans un monde de l'écrit. Encore une fois, c'est une manière de valoriser la vie populaire et urbaine. Si cette littérarité nous habite naturellement et quotidiennement, le quotidien devient par conséquent un sujet valable pour la littérature. Bien sûr, le cahier ne s'écrit pas d'une manière si naïve de sorte qu'il faut également voir dans cette prolifération intertextuelle une tentative de brouiller les distinctions entre les littératures occidentale et africaine, dont les grands livres composent la littérature-monde, quelles que soient leurs origines géographiques.

Cette tendance à l'harmonisation est l'une des plus constantes du livre. C'est souvent aux moments les plus «grotesques» que Verre Cassé insère des références littéraires les plus sublimes. Une évocation exemplaire de cette tendance a lieu après que Verre Cassé part à la recherche d'une prostituée afin de retrouver sa virilité perdue. Au milieu de cette scène surgit une référence à la poésie de Baudelaire : 
Alice aux jambes maigres et arquées a ôté la ficelle qui me servait de ceinture, elle a déboutonné mon pantalon décati, elle a plongé dedans sa main aux doigts distordus, elle a trouvé mon truc plus que contracté, « je vais m’en occuper, chéri, ton machin va être debout comme si tu avais encore vingt ans, j'ai l'habitude, crois-moi », et elle a commencé à convoquer ses souvenirs de jeune prostituée lorsque ses mains pouvaient encore faire perdre la tête à un traîne-misère au bord du suicide, mais ses gestes étaient veules comme ceux d'un albatros capturé en haute mer par des hommes d'équipage qui souhaitent s'amuser, et donc la vieille bique pétrissait plus qu'elle ne caressait [...] (109-10).

Le lecteur peut facilement identifier la référence à l'un des plus beaux poèmes de Baudelaire, «L'Albatros ». Verre Cassé mêle donc une scène conventionnellement présentée comme extrêmement vulgaire (bien que partageant plusieurs aspects de l'esthétique charnelle de Baudelaire) à ce qui est conventionnellement considéré comme la beauté de la poésie baudelairienne. Cette référence a une double fonction: Verre Cassé se met à la place de l'albatros, il est aussi maladroit que cet animal capturé. Son esprit essaie d'atteindre les hauteurs de l'expression, mais il reste empêché sur terre. Or, le discours hybride de Verre Cassé montre que rester sur terre c'est peut-être un acte solidaire avec son milieu, avec son espace au bar, avec ses compatriotes. Rester sur terre, c'est revaloriser tout ce qui l'environne et c'est rester en contact étroit avec son espace.

En effet, le lecteur ne peut même pas distinguer entre le jeu intertextuel et «l'écriture normale » de Verre Cassé. On a l’impression que Verre Cassé (pour ne pas dire Mabanckou lui-même) vient de ramasser toute une bibliothèque et qu'il s'efforce d'en mettre l'intégralité dans le livre ${ }^{11}$. Le jeu intertextuel étonne aussi par son éclectisme géographique et culturel, qui fait voyager le lecteur averti de la Martinique (Patrick Chamoiseau) à la Guinée (Camara Laye), de la Côte d'Ivoire (Ahmadou Kourouma) au Sénégal (Aminata Sow Fall), pour ne citer qu'une infime partie des références ${ }^{12}$. Ce "goût effréné » (Nissim 207) de l'intertextualité montre la maîtrise hors-norme de Verre Cassé sur l'art de l'écriture, même s'il déclare qu'il n'est pas écrivain : «j'ai dit à L'Escargot entêté en guise de conclusion que malheureusement j'étais pas écrivain, que je ne pouvais pas l'être, que moi je ne faisais qu'observer et parler aux bouteilles »

11 Nissim consacre une page et demie de son article en relevant les titres des œuvres « citées » directement ou indirectement dans le livre, un effort qui n'en arrive pas moins qu’à relever « quelques-uns » de tous les livres mentionnés par Verre Cassé.

${ }^{12}$ Voir l'étude de Kathryn Batchelor, « Postcolonial Intertextuality and Translation Explored through the Work of Alain Mabanckou » (2013), pour une analyse soignée du problème de la traduction des références intertextuelles chez Mabanckou dans la version en langue anglaise de son roman. De nombreuses références venant d'espaces non-hexagonaux ne sont pas traduites tandis que la plupart des intertextes français sont reconnus. 
(162). La modestie est fausse, mais elle permet surtout une mise en contact de plusieurs espaces à travers les langages populaire et littéraire.

\section{Une poétique des espaces}

Il est également important de noter que même si Verre Cassé donne une voix à un quartier démuni, il ne reste pas pour autant replié sur lui-même. En effet, Verre Cassé, comme nous tous, aime lire la littérature justement pour se laisser emporter par la page écrite : « je peux toutefois noter sur cette page que, sans me vanter, d'une manière ou d'une autre, j'ai voyagé à travers le monde, je ne voudrais pas qu'on me prenne pour un gars qui ignore les choses qui se passent hors de sa terre natale, je n'accepterais pas un tel raccourci [...] je ne rencontrais alors aucune frontière au cours de mes odyssées » (170-1). Verre Cassé devient un acteur de «la fratrie francophone» («Le chant»56) que Mabanckou appellerait de ses vœux dans sa contribution au manifeste Pour une littératuremonde. Cet écrivain modeste se montre engagé dans le monde littéraire, ce qui lui permet de «brise $[\mathrm{r}]$ les frontières » («Le chant» 56) pour voyager vers d'autres espaces avec le pouvoir de son langage à la fois populaire et littéraire. Verre Cassé est en train d'esquisser les prémisses d'une nouvelle théorie littéraire que nous pourrions qualifier de «poétique des espaces».

Cette théorie littéraire sera approfondie après que L'Escargot entêté convoque Verre Cassé pour écrire un livre. Verre Cassé donne une longue réponse véhémente (pleine d'intertextes) dont voici quelques extraits : "c'était simple pour moi de m'exprimer [...] je les dirais avec des mots à moi [...] j'écrirais comme des mots me viendraient [...] ce bazar c'est la vie, entrez donc dans ma caverne, y a de la pourriture, y a des déchets, c'est comme ça que je conçois la vie » (160-2). Enfin, Verre Cassé se fait un plaisir d'écrire : "c'est quand je m'abandonne, quand j'oublie qu'une mission m'avait été confiée que je me sens vraiment dans mon assiette puisque je peux sauter, cabrioler, parler à un lecteur autre » (164). La poétique est inséparable du langage particulier, mais un langage qui invite « à s'ouvrir à l'autre sans nous perdre » (Le monde 64).

La poétique des espaces que développe Mabanckou se relie de manière forte aux idées d'Édouard Glissant sur le Tout -Monde et la Relation. Le « voyage en littérature » de Verre Cassé correspond à la vision glissantienne d'un mouvement immobile : «L'idée du monde s'autorise de l'imaginaire du monde, des poétiques entremêlées qui me permettent de deviner en quoi mon lieu conjoint à d'autres, en quoi sans bouger, il s'aventure ailleurs, et comment il m'emporte dans ce mouvement immobile » (Traité 120). Même si Verre Cassé reste «immobile », il est capable de « s'aventurer » à travers un imaginaire et un langage complexes. Il montre l'importance de l'emportement littéraire et langagier pour « entremêler » 
plusieurs espaces du monde. Verre Cassé montre la possibilité, voire la nécessité de relever l'importance de notre espace particulier sans jamais refuser la diversité des autres espaces globaux: "j’appelle Poétique de la Relation ce possible de l'imaginaire qui nous porte à concevoir la globalité [...] en même temps qu'il nous permet d'en relever quelque détail, et en particulier de chanter notre lieu [...]» (Traité 22); " la Relation est ici entendue comme la quantité réalisée de toutes les différences $d u$ monde» (Relation 42). Pour Mabanckou, il s'agit de «ne pas m'enfermer... mais de prêter plutôt l'oreille à la rumeur du monde » (Le monde 11), une ouverture et une disponibilité à la diversité culturelle et littéraire que l'auteur entame dans Verre Cassé et qui trouvent son point culminant dans son « autobiographie capricieuse », Le monde est mon langage. Si Verre Cassé réussit sa mission grâce à un « voyage en littérature », c'est parce que Mabanckou trouve dans « les lieux, les voyages, les auteurs et l'écriture... un moyen de féconder un humanisme où l'imaginaire serait aussi bariolé que l'arc-en-ciel et nous pousserait à nous remettre en question » (Le monde 12).

À la fin du livre, le récit semble se précipiter, et les références et hommages littéraires se multiplient (en quelques pages il cite Césaire, García Márquez, Tagore, Laferrière, Hugo, Baudelaire, et Hemingway parmi d'autres). Il se dit prêt à tout abandonner: «je me laisse aller comme une immondice qui suit le courant d'un fleuve détourné » (174). Que faut-il comprendre dans la mort par noyade de Verre Cassé? Dans un sens, Verre Cassé a réussi sa mission de conteur de la singularité de son espace. Il se jette dans la rivière Tchinouka, ce qui crée un parallèle avec sa mère noyée. La bibliothèque ne brûle donc pas, mais elle se noie avec Verre Cassé. Or, sa connaissance littéraire est préservée dans le cahier, confié à la fin à Holden, lui-même un personnage du roman de Salinger. Verre Cassé veut que le cahier soit lu, mais il ne peut plus se limiter à un seul espace sur terre. La rivière rejoint le fleuve qui se jette dans l'océan pour atteindre le monde. On passe d'un espace local à un espace global. Par ces deux actions, la remise du cahier et sa mort par noyade, Verre Cassé accomplit sa mission littéraire.

Enfin, dans ce livre où le langage de tous les jours vient se mêler au langage de la littérature canonisée, nous avons une réponse à cette question éternelle : «Qu'est-ce que la littérature? ». La littérature, c'est le langage qui s'ouvre au chaos du monde, qui trouve de la beauté aux espaces pauvres et aux quartiers les plus «crasseux ». La réussite de Verre Cassé c'est enfin de redonner à la littérature une «vie derrière les mots» (160), une vie quotidienne. C'est le langage qui est la vraie force du livre. Le langage qui nous permet d'établir une relation étroite avec notre milieu, notre espace local tout en prenant part au grand monde, l'espace global. Lire cette littérature «devient alors à la fois un enracinement, un appel dans la nuit et une oreille tendue vers l'horizon » (Le 
monde 11). Comme Glissant, Verre Cassé incarne « la passion nouvelle de voir réaliser cette totalité monde, sans en excepter la plus inaperçue des composantes » (Relation 29). Pour reprendre l'idée d'écoumène proposée par Berque, il semble que Mabanckou réussisse à la dépasser. Oui, le livre montre les relations complexes entre les êtres humains et l'espace qui les entoure, mais il faut ajouter un autre espace à ces relations, un espace « plus étendu, plus éclaté, plus bruyant, c'est-à-dire le monde » («Le chant » 65). L'écoumène, régional au début, devient universel. Voilà comment Mabanckou crée une poétique des espaces - une poétique qui comprend toute la complexité du monde. 


\section{Bibliographie}

Augé, Marc. Non-lieux : Introduction à une anthropologie de la surmodernité. Seuil, 1992. Batchelor, Kathryn. "Postcolonial Intertextuality and Translation Explored through the Work of Alain Mabanckou ». Intimate Enemies: Translation in Francophone Contexts, edité par Kathryn Batchelor \& Claire Bisdorff, Liverpool University Press, 2013, pp. 196-215.

Berque, Augustin. Écoumène : Introduction à l'étude des milieux humains. Belin, 2000.

Blanc, Nathalie, Chartier, Denis, et Pughe, Thomas (éds.). «Littérature \& écologie : vers une écopoétique », numéro spécial d’Écologie \& Politique, n'36, 2008.

Collot, Michel. « Pour une géographie littéraire ». Fabula-LbT, n 8 , « Le partage des disciplines », mai 2011, www.fabula.org/lht/8/collot.html. Consulté le 2 janvier 2019.

Dardel, Éric, L'Homme et la terre: nature de la réalité géographique. Presses Universitaires de France, 1952.

Derrida, Jacques. Le monolinguisme de l'autre, ou, la prothèse d'origine, Galilée, 1996.

Genette, Gérard. «La Littérature et l'espace. » Figures II. Seuil, 1969, pp. 43-48.

Glissant, Édouard. Philosophie de la relation : poésie en étendue. Gallimard, 2009.

---. Traité du Tout-monde. Gallimard, 1997.

Mabanckou, Alain. «Le chant de l'oiseau migrateur. » Pour une littérature-monde, édité par Michel Le Bris \& Jean Rouaud, Gallimard, 2007, pp. 55-66.

---. Le monde est mon langage. Grasset, 2016.

---. Verre Cassé. Seuil, 2005.

Mambenga-Ylagou, Frédéric. «Français et imaginaire populaires ou la poétique du social urbain dans la littérature africaine francophone.» Discours et écritures dans les sociétés en mutation, édité par Manuel Bengoéchéa, Dephine Chaume, Claire Riffard, et Katerina Spiropoulou, Paris, L'Harmattan, 2007, pp. 135-154.

Nissim, Liana. «Le Roman africain du troisième millénaire. Un exemple : Alain Mabanckou ou le miracle de l'écriture. » Où va la francophonie au début du troisième millénaire? Actes du colloque de Bari, 4-5 Mai 2005, édité par Giovanni Dotoli, PU Paris-Sorbonne, 2005, pp. 199-216.

Posthumus, Stéphanie. «Vers une écocritique française : le contrat naturel de Michel Serres. » Mosaic, vol. 44, n², 2011, pp. 85-100.

Prieto, Eric. "Geocriticism Meets Ecocriticism: Bertrand Westphal and Environmental Thinking." Ecocriticism and Geocriticism. Overlapping Territories in Environmental and Spatial Literary Studies, édité par Robert T. Tally, Christine M. Battista, Palgrave Macmillan, 2014, pp. 19-36.

Tally, Robert T. Spatiality. Routledge, 2013.

Westphal, Bertrand, La géocritique : réel, fiction, espace. Minuit, 2007. 
---. «Pour une approche géocritique des textes». La Géocritique mode d'emploi, Limoges, PULIM, collection « Espaces Humains », 2000, pp. 9-40. 